\title{
Redução do Mecanismo de Reações para Fluxos Quimicamente Não-Equilibrados em Tubeiras Supersônicas
}

\author{
A. P. Spilimbergo ${ }^{1}$ \\ Departamento de Ciências Exatas e Engenharias, UNIJUÍ, Ijuí, RS \\ V. G. Krioukov ${ }^{2}$ e R. L. Iskhakova ${ }^{3}$ \\ Department of Automobile Engines and Service, KNITU-KAI, Kazan, Rússia
}

\begin{abstract}
Resumo. Atualmente, o cálculo de fluxos quimicamente não-equilibrados em tubeiras é baseado na cinética química detalhada. Neste trabalho propõem-se o procedimento de redução dos mecanismos de reações que é constituído pelos métodos DRGEP e de Engajamento. Este procedimento foi aplicado ao problema do cálculo de fluxos não-equilibrados dos produtos de combustão dos bipropelentes: "Querosene $+\mathrm{O}_{2}$ " e " $\mathrm{N}_{2} \mathrm{O}_{4}+\mathrm{C}_{2} \mathrm{H}_{8} \mathrm{~N}_{2}$ ". Como resultado foi atingida uma redução significativa do mecanismo inicial de reações com pequenos erros na previsão das características de fluxos.
\end{abstract}

Palavras-chave. Tubeira Supersônica, Redução de Mecanismo, Cinética Química.

\section{Introdução}

O cálculo dos processos quimicamente não-equilibrados em tubeiras supersônicas é um problema tradicional, e o interesse neste problema não diminui devido a pesquisas de novos propelentes e de desenvolvimento de novos esquemas de motores de foguetes $[2,5]$. Atualmente, os modelos matemáticos desses fluxos baseiam-se na cinética química detalhada. Os mecanismos das transformações químicas (usados nessa abordagem) são constituídos por muitas reações (100 ou mais), o que leva a um grande volume de cálculo. Mas, ao mesmo tempo a maioria das reações influi minimamente nas características de combustão e para determinar tais reações desenvolvem-se vários métodos de redução: métodos de análise das velocidades das reações $[7,8]$; métodos que utilizam autovalores $[6$, 7]; métodos que operam com dados preliminarmente tabulados [4, 6], etc. Mas, todos estes métodos (e suas combinações) são aplicados somente em esquemas simples de combustão: "Batch Reactor" (BR), "Perfectly Stirred Reactor" (PSR), "Plug Flow Reactor" (PFR). Neste trabalho o algoritmo proposto em [6] é aplicado a tubeira supersônica (cujo esquema de combustão é mais complexo).

Para tubeiras o problema de redução do mecanismo tem as seguintes particularidades:

\footnotetext{
${ }^{1}$ patspi@unijui.edu.br

22vkrujkov@mail.ru

${ }^{3}$ vkrujkov@kai.ru
} 
- na entrada da tubeira o meio reagente é quimicamente equilibrado e após, a medida que ocorre o movimento, a composição é alterada de acordo com o mecanismo de reações; - a característica mais importante do fluxo não-equilibrado é o coeficiente de perda do impulso específico devido ao desequilíbrio químico $\left(\xi_{d q}\right)$, e com a redução do mecanismo o erro deste coeficiente deve permanecer minimal.

\section{Técnica de Cálculo do Fluxo Não-Equilibrado e o Algo- ritmo de Redução}

O fluxo unidimensional não-equilibrado é descrito pelas equações [4] a seguir.

(a) Equação da Cinética Química, na forma exponencial:

$$
\begin{gathered}
\frac{d \gamma_{i}}{d x}=\frac{1}{V_{g}}\left(-e^{\gamma_{i}} \sum_{j} \nu_{i j} \Omega_{j}+\sum_{q} \sum_{j} \nu_{q j} \Omega_{j}\right) \equiv f_{i}^{\gamma} \\
\Omega_{j}=k_{j}\left(\frac{P}{R_{o} T}\right)^{\bar{m}_{j}} \exp \left(-\sum_{p} n_{p j} \gamma_{p}\right) ; \quad \bar{m}_{j}=m_{j}+\Sigma n_{p j}-1 ; \quad \gamma_{i}=-\ln r_{i} ; \\
\nu_{i j}=\nu_{i s}^{\prime \prime}-\nu_{i s}^{\prime} ; \quad n_{i j}=\nu_{i s}^{\prime} ; \quad j=s ; \quad s=1, \ldots, m_{c} \\
\nu_{i j}=\nu_{i s}^{\prime}-\nu_{i s}^{\prime \prime} ; \quad n_{i j}=\nu_{i s}^{\prime \prime} ; \quad j=s+m_{c} ; \quad s=1, \ldots, m_{c}
\end{gathered}
$$

com $i, p, q=1, \ldots, n_{c} ; j=1, \ldots, 2 m_{c}$ e $\nu_{i s}^{\prime}$ e $\nu_{i s}^{\prime \prime}$ coeficientes estequiométricos no conjunto de reações reversíveis:

$$
\sum_{i} \nu_{i s}^{\prime} B_{i} \Longleftrightarrow \sum_{i} \nu_{i s}^{\prime \prime} B_{i} ; \quad s=1, \ldots, m_{c}
$$

onde $B_{i}$ é o símbolo da $i$-ésima substância; $m_{j}$ é o índice de participação na $j$-ésima reação da partícula catalítica $M\left(m_{j}=1\right.$ se a partícula $M$ participa na $j$-ésima reação e $m_{j}=0$ de maneira oposta); $k_{j}$ é a constante de velocidade da $j$-ésima reação ; $n_{c}$ e $m_{c}$ correspondem ao número de espécies e reações no meio reagente; $R_{o}$ a constante universal de gás; $P$ a pressão e $T$ a temperatura.

(b) Equação do Movimento e da Energia:

$$
\frac{d V_{g}}{d x}=\frac{R_{o} T}{V_{g} \sum_{i} r_{i} \mu_{i}} \varphi^{\prime}(x) ; \quad h_{o c}-\frac{V_{g}^{2}}{2}=\frac{\sum_{i} H_{i} r_{i}}{\sum_{i} r_{i} \mu_{i}}
$$

onde: $h_{o c}$ é a entalpia mássica na entrada da tubeira; $V_{g}$ a velocidade do gás; $r_{i}, \mu_{i}$ e $H_{i}$ são fração molar, massa molecular e entalpia molar da $i$-ésima substância.

(c) Equação da Dependência $P=P(x)$ :

$$
\varphi(x)=-\ln \left(P / P_{o c}\right)
$$

onde $P_{o c}$ é a pressão na entrada da tubeira. 
A Figura 1 mostra a alteração geral das características do fluxo ao longo da tubeira, onde: 1 - câmara de combustão; 2 - tubeira; oc - índice da secção de entrada; M - índice da secção mínima e a - saída da tubeira.

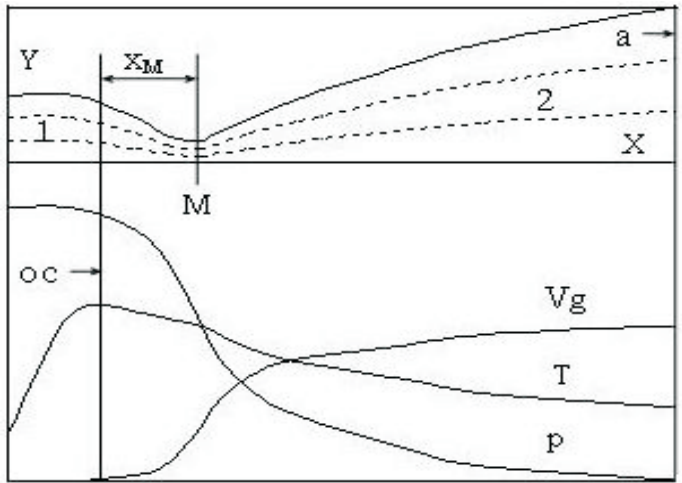

Figura 1: Alteração das características $V_{g}, T$ e $P$ ao longo da tubeira [1].

Para determinar o coeficiente $\xi_{d q}$ é necessário aplicar modelos de fluxo quimicamente equilibrado, que são amplamente conhecidos [1] e fluxo quimicamente não-equilibrado apresentado pelas Eq.(1)-(6). Depois disso, é necessário calcular os impulsos específicos: de equilíbrio $\left(I_{1}^{e s}\right)$ e de não-equilíbrio $\left(I_{2}^{e s}\right)$ por $(7)$.

$$
I_{z}^{e s}=V_{g z a}+P_{z a} /\left(\rho_{z a} . V_{g z a}\right) ; \quad z=1 ; 2
$$

onde $V_{g z a}$ é a velocidade; $P_{z a}$ a pressão e $\rho_{z a}$ a densidade dos produtos de combustão na saída da tubeira e, em seguida, encontrar o coeficiente de perda do impulso específico dado em (8).

$$
\xi_{d q}=\left(I_{1}^{e s}-I_{2}^{e s}\right) / I_{1}^{e s}
$$

O algoritmo de redução do mecanismos de reações utiliza as seguintes etapas:

- inicialmente constitui-se o mecanismo original (ou $C$-mecanismo) de reações, que deve ser reduzido, também é necessário definir a área de variação dos parâmetros envolvidos no mecanismo de redução;

- o mecanismo de redução para um ponto dos parâmetros é chamado local ( $L$-mecanismo) e para uma área de alteração dos parâmetros é indicado por $G$-mecanismo. Geralmente o $G$-mecanismo é formado pelo acoplamento dos $L$-mecanismos;

- especificação de um conjunto de substâncias prescritas que durante a redução não podem ser removidas do mecanismo diferentemente de outras substâncias (sondadas);

- escolha dos indicadores de redução (limiares) e para cada método usado no algoritmo é definido o seu próprio limiar. Quanto maior o valor do limiar, maior será o grau da redução do mecanismo original, mas também maior o erro de cálculo (previsão).

Em [8] foi apresentado o algoritmo que é constituído pelo método DRGEP (Directed Relation Graph Error Propagation) [7] e pelo método de Engajamento [8]. Diferentemente de outros algoritmos de redução $[3,6,7,9]$ esse procedimento requer um pequeno volume computacional e reduz efetivamente o mecanismo original. 


\section{Exemplos de Redução dos Mecanismo de Reações para os Fluxos em Tubeiras}

Para a validação do procedimento proposto foram formados $G$-mecanismos para tubeiras perfiladas para a seguinte área de alteração dos parâmetros:

$$
\alpha_{o x}=0.7, \ldots, 1.2 ; \quad P_{o c}=20, \ldots, 100 a t m \quad r_{M}=0.006, \ldots, 0.06 m
$$

onde $\alpha_{o x}$ é o coeficiente de excesso do oxidante e $r_{M}$ o raio da seção mínima da tubeira. A) Bipropelente "Querosene $+\mathrm{O}_{2}$ ". O mecanismo inicial foi constituído por 16 substâncias e 47 reações, retiradas de [3]. Foram selecionadas como substâncias prescritas: $\mathrm{CO}_{2}, \mathrm{O}_{2}$, $\mathrm{H}_{2} \mathrm{O}$ e $\mathrm{CO}$. Inicialmente, no regime de fluxo com parâmetros: $\alpha_{o x}=1.0 ; P_{o c}=20 \mathrm{~atm}$; $r_{M}=0.006 m$ (regime básico) foram estabelecidos os limiares $\zeta_{d}$ (para o método DRGEP) e $\zeta_{a o}$ (para o método de Engajamento) para toda área de abrangência do $G$-mecanismo, conforme (9). A redução significativa do mecanismo inicial é alcançada já para pequenos limiares $\zeta_{L} \leq 0.02$ (Tabela 1 ) e no $L$-mecanismo permanecem 9 substâncias: $H, H_{2}, O$, $\mathrm{O}_{2}, \mathrm{CO}_{2}, \mathrm{H}_{2} \mathrm{O}, \mathrm{CO}, \mathrm{OH}$ e $\mathrm{HCO}$.

Tabela 1: Características do fluxo na saída da tubeira (regime básico) em função dos limiares.

\begin{tabular}{|c|c|c|c|c|c|c|c|c|}
\hline Mecanismo & $B_{i}(L)$ & $R_{f}(L)$ & $T_{a}\left({ }^{\circ} \mathrm{K}\right)$ & $\mathrm{CO}_{2}$ & $\mathrm{O}_{2}$ & $\mathrm{H}_{2} \mathrm{O}$ & $\mathrm{CO}$ & $\xi_{X_{M}}(\%)$ \\
\hline$C$-mecanismo & 16 & 47 & 1515 & 0.2875 & 0.0933 & 0.3447 & 0.1577 & 5.213 \\
\hline$\zeta_{L}=0.01$ & 9 & 13 & 1515 & 0.2875 & 0.0933 & 0.3447 & 0.1577 & 5.215 \\
\hline$\zeta_{L}=0.02$ & 9 & 13 & 1515 & 0.2875 & 0.0933 & 0.3447 & 0.1577 & 5.215 \\
\hline$\zeta_{L}=0.04$ & 8 & 10 & 1501 & 0.2859 & 0.0941 & 0.3435 & 0.1583 & 5.329 \\
\hline
\end{tabular}

Para a Tabela 1 foi considerado que $\zeta_{d}=\zeta_{a o}=\zeta_{L}$, que as concentrações das substâncias são dadas em frações molares e que $B_{i}(L)$ e $R_{f}(L)$ correspondem ao número de substâncias e reações nos $L$-mecanismos, respectivamente.

Ao longo do comprimento da tubeira os erros também são quase invisíveis, como pode ser visto na Fig. 2. Assim, o limiar $\zeta_{G}=\zeta_{L}=0.02$ foi estabelecido para outros regimes de fluxos não-equilibrados. Depois foram selecionados cerca de 40 pontos $\left\{\alpha_{o x} ; P_{o c} ; r_{M}\right\}$ na área (9) e para cada ponto foi realizado o cálculo do fluxo supersônico e foi gerado um $L$-mecanismo. Alguns resultados para esses mecanismos são mostrados na Tabela 2, onde: $T_{a}(C), T_{a}(L), \xi_{d q}(C)$ e $\xi_{d q}(L)$ são características na saída de tubeira obtidas pelo $C$-mecanismo e pelo $L$-mecanismo. Nas colunas de 2 a 7 são dadas as características do $L$-mecanismo para os pontos $P_{o c}=20 \mathrm{~atm} ; r_{M}=0.006 \mathrm{~m}$, sendo $\alpha_{o x}$ variando de 0.7 a 1.2 com passo $\Delta \alpha_{o x}=0.1$, e nas colunas 8 e 9 estão mostradas as mesmas características para os pontos:

$$
\begin{aligned}
& P 1=\left\{\alpha_{o x}=1.0 ; \quad P_{o c}=100 a t m ; \quad r_{M}=0.006 m\right\} \\
& P 2=\left\{\alpha_{o x}=1.0 ; \quad P_{o c}=100 a t m \quad \text { e } \quad r_{M}=0.06 m\right\}
\end{aligned}
$$

Como pode ser visto cada $L$-mecanismo contém 9 substâncias acima mencionadas, mas com diferentes números de reações (de 11 a 14 reações). A temperatura de saída da tubeira 


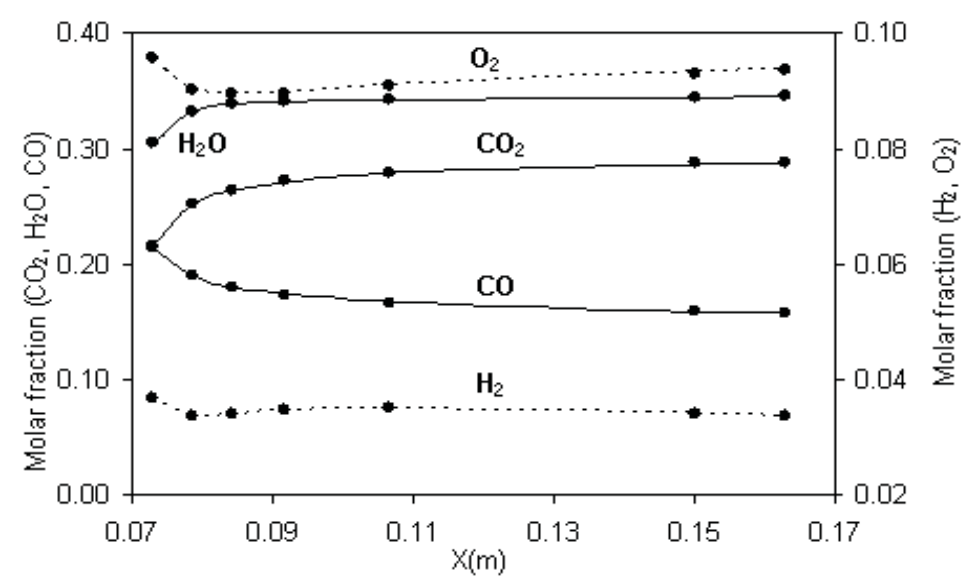

Figura 2: Alteração da composição e da temperatura ao longo da tubeira (regime básico) para o $C$-mecanismo $(\bullet)$ e $L$-mecanismo $\left(\zeta_{L}=0.02\right)$ para o bipropelente "Querosene $+\mathrm{O}_{2}$ ".

Tabela 2: Comparação das características dos fluxos para os produtos de combustão do bipropelente "Querosene $+\mathrm{O}_{2}$ ", utilizando $C$ e $L$ mecanismos $\left(\zeta_{G}=0.02\right)$.

\begin{tabular}{|c|c|c|c|c|c|c|c|c|}
\hline & \multicolumn{6}{|c|}{$P_{o c}=20 a t m ; r_{M}=0.006 \mathrm{~m}$} & $(P 1)$ & $(P 2)$ \\
\hline$\alpha_{o x}$ & 0.7 & 0.8 & 0.9 & 1.0 & 1.1 & 1.2 & 1.0 & 1.0 \\
\hline$B_{i}(L)$ & 9 & 9 & 9 & 9 & 9 & 9 & 9 & 9 \\
\hline$R_{f}(L)$ & 11 & 12 & 13 & 13 & 14 & 12 & 13 & 11 \\
\hline$T_{a}(C)^{o} K$ & 1258 & 1436 & 1509 & 1515 & 1493 & 1461 & 1850 & 2055 \\
\hline$T_{a}(L)^{o} K$ & 1258 & 1435 & 1510 & 1515 & 1493 & 1461 & 1851 & 2055 \\
\hline$\xi_{d q}(C) \%$ & 2.372 & 4.119 & 5.037 & 5.213 & 5.310 & 5.335 & 2.362 & 1.080 \\
\hline$\xi_{d q}(L) \%$ & 2.383 & 4.163 & 4.983 & 5.215 & 5.261 & 5.354 & 2.369 & 1.080 \\
\hline
\end{tabular}

$T_{a}(L)$ é prevista com erro muito pequeno. Conjugando todos os $L$-mecanismos gerados, obtém-se o $G$-mecanismo reduzido (Tabela 3 ) para toda a área (9).

De acordo com a regra: "O volume de cálculo é proporcional ao número de reações e ao cubo do número de substâncias" $[6,9]$, pode ser estimado o indicador da relação dos volumes computacionais entre $C$ e $G$ mecanismos:

$$
\eta(G, C)=\frac{\operatorname{Volume}(C)}{\operatorname{Volume}(G)}=\left(\frac{47}{15}\right) \times\left(\frac{16}{9}\right)^{3} \approx 17.6
$$

B) Bipropelente " $\mathrm{N}_{2} \mathrm{O}_{4}+\mathrm{C}_{2} \mathrm{H}_{8} \mathrm{~N}_{2}$ ". Este sistema reagente é mais complexo e as substâncias prescritas foram: $\mathrm{CO}_{2}, \mathrm{O}_{2}, \mathrm{H}_{2} \mathrm{O}, \mathrm{CO}$ e $\mathrm{N}_{2}$. O mecanismo original foi constituído por 26 substâncias e 80 reações, retiradas de [3]. Inicialmente de acordo com os resultados dos cálculos para o regime básico (por analogia com o exemplo A) foram estabelecidos limiares $\zeta_{G}=\zeta_{L}=\zeta_{d}=\zeta_{a o}=0.02$ para toda área que abrange o $G$-mecanismo.

A formação do $G$-mecanismo foi realizada para a mesma área (9) de alterações dos parâmetros: $\alpha_{o x}, P_{o c}$ e $r_{M}$. Para isso foram selecionados 40 pontos $\left\{\alpha_{o x}, P_{o c}, r_{M}\right\}$ e para 
Tabela 3: $G$-mecanismo para prever as características dos fluxos dos produtos de combustão do bipropelente "Querosene $+\mathrm{O}_{2}$ " para a área $(9)\left(\zeta_{G}=0.02\right)$.

\begin{tabular}{|c|c|c|}
\hline Reação & Reação & Reação \\
\hline $\mathrm{H}_{2}+\mathrm{OH}=\mathrm{H}_{2} \mathrm{O}+\mathrm{H}$ & $2 \mathrm{O}+\mathrm{M}=\mathrm{O}_{2}+M$ & $\mathrm{CO}+\mathrm{O}+\mathrm{M}=\mathrm{CO}_{2}+\mathrm{M}$ \\
\hline $\mathrm{O}+\mathrm{H}_{2}=\mathrm{OH}+\mathrm{H}$ & $\mathrm{H}+\mathrm{M}+\mathrm{OH}=\mathrm{H}_{2} \mathrm{O}+\mathrm{M}$ & $\mathrm{CO}_{2}+\mathrm{H}_{2}=\mathrm{OH}+\mathrm{HCO}$ \\
\hline $\mathrm{H}+\mathrm{O}_{2}=\mathrm{OH}+\mathrm{O}$ & $\mathrm{O}+\mathrm{H}+\mathrm{M}=\mathrm{OH}+\mathrm{M}$ & $\mathrm{CO}_{2}+\mathrm{H}=\mathrm{O}+\mathrm{HCO}$ \\
\hline $2 \mathrm{OH}=\mathrm{H}_{2} \mathrm{O}+\mathrm{O}$ & $\mathrm{CO}+\mathrm{O}_{2}=\mathrm{CO}_{2}+\mathrm{O}$ & $\mathrm{HCO}+\mathrm{M}=\mathrm{H}+\mathrm{CO}+\mathrm{M}$ \\
\hline $2 \mathrm{H}+\mathrm{M}=\mathrm{H}_{2}+\mathrm{M}$ & $\mathrm{CO}+\mathrm{OH}=\mathrm{CO}_{2}+\mathrm{H}$ & $\mathrm{HCO}+\mathrm{H}=\mathrm{CO}+\mathrm{H}_{2}$ \\
\hline
\end{tabular}

cada um deles foi gerado um $L$-mecanismo.

Conjugando todos os $L$-mecanismos foi formado um $G$-mecanismo, constituído por 27 reações mostradas na Tabela 4 e pelas substâncias: $\mathrm{H}, \mathrm{H}_{2}, \mathrm{O}, \mathrm{O}_{2}, \mathrm{CO}_{2}, \mathrm{H}_{2} \mathrm{O}, \mathrm{CO}, \mathrm{OH}$, $\mathrm{N}, \mathrm{N}_{2}, \mathrm{NO}, \mathrm{NH}, \mathrm{HCO}, \mathrm{HNO}$ e $\mathrm{N}_{2} \mathrm{O}$.

Tabela 4: $G$-mecanismo para prever as características dos fluxos dos produtos de combustão do bipropelente " $\mathrm{N}_{2} \mathrm{O}_{4}+\mathrm{C}_{2} \mathrm{H}_{8} \mathrm{~N}_{2}$ " para a área (9) $\left(\zeta_{G}=0.02\right)$.

\begin{tabular}{|c|c|c|}
\hline Reação & Reação & Reação \\
\hline $\mathrm{H}_{2}+\mathrm{OH}=\mathrm{H}_{2} \mathrm{O}+\mathrm{H}$ & $\mathrm{CO}_{2}+\mathrm{H}_{2}=\mathrm{OH}+\mathrm{HCO}$ & $\mathrm{HNO}+\mathrm{OH}=\mathrm{NO}+\mathrm{H}_{2} \mathrm{O}$ \\
\hline $\mathrm{O}+\mathrm{H}_{2}=\mathrm{OH}+\mathrm{H}$ & $\mathrm{CO}_{2}+\mathrm{H}=\mathrm{O}+\mathrm{HCO}$ & $\mathrm{N}+\mathrm{OH}=\mathrm{NO}+\mathrm{H}$ \\
\hline $\mathrm{H}+\mathrm{O}_{2}=\mathrm{OH}+\mathrm{O}$ & $\mathrm{HCO}+\mathrm{M}=\mathrm{H}+\mathrm{CO}+\mathrm{M}$ & $\mathrm{N}_{2} \mathrm{O}+\mathrm{H}=\mathrm{N}_{2}+\mathrm{OH}$ \\
\hline $2 \mathrm{OH}=\mathrm{H}_{2} \mathrm{O}+\mathrm{O}$ & $\mathrm{HCO}+\mathrm{H}=\mathrm{CO}+\mathrm{H}_{2}$ & $\mathrm{HNO}+\mathrm{H}=\mathrm{H}_{2}+\mathrm{NO}$ \\
\hline $2 \mathrm{H}+\mathrm{M}=\mathrm{H}_{2}+\mathrm{M}$ & $\mathrm{CO}+\mathrm{O}+\mathrm{M}=\mathrm{CO}_{2}+\mathrm{M}$ & $\mathrm{NH}+\mathrm{NO}=\mathrm{N}_{2} \mathrm{O}+\mathrm{H}$ \\
\hline $2 \mathrm{O}+\mathrm{M}=\mathrm{O}_{2}+\mathrm{M}$ & $\mathrm{N}+\mathrm{NO}=\mathrm{N}_{2}+\mathrm{O}$ & $\mathrm{NH}+\mathrm{O}_{2}=\mathrm{HNO}+\mathrm{O}$ \\
\hline $\mathrm{H}+\mathrm{M}+\mathrm{OH}=\mathrm{H}_{2} \mathrm{O}+\mathrm{M}$ & $\mathrm{N}+\mathrm{O}_{2}=\mathrm{NO}+\mathrm{O}$ & $\mathrm{HO}+\mathrm{M}=\mathrm{H}+\mathrm{NO}+\mathrm{M}$ \\
\hline $\mathrm{O}+\mathrm{H}+\mathrm{M}=\mathrm{OH}+\mathrm{OH}$ & $\mathrm{NH}+\mathrm{H}=\mathrm{N}+\mathrm{H}_{2}$ & $\mathrm{NH}+\mathrm{OH}=\mathrm{N}+\mathrm{H}_{2} \mathrm{O}$ \\
\hline $\mathrm{CO}+\mathrm{OH}=\mathrm{CO}_{2}+\mathrm{H}$ & $\mathrm{N}_{2} \mathrm{O}+\mathrm{M}=\mathrm{N}_{2}+\mathrm{O}+\mathrm{M}$ & $\mathrm{NH}+\mathrm{OH}=\mathrm{HNO}+\mathrm{H}$ \\
\hline
\end{tabular}

\section{Conclusões}

1. Neste trabalho foi proposto um procedimento para reduzir o mecanismo original (redundante) a um tamanho aceitável, fornecendo, na previsão das características do fluxo em tubeiras supersônicas, um erro controlado.

2. Este procedimento que contém os métodos DRGEP e de Engajamento, foi introduzido no código de cálculo dos processos quimicamente não-equilibrados em tubeiras.

3. Aplicando este procedimento para uma ampla área de alterações dos parâmetros $\left(\alpha_{o x}=\right.$ $\left.0.7, \ldots, 1.2 ; P_{o c}=20, \ldots, 100 a t m ; r_{M}=0.006, \ldots, 0.06 m\right)$ foram obtidos os seguintes $G-$ mecanismos (com pequenos erros nas caraterísticas do fluxo):

- para o bipropelente "Querosene $+\mathrm{O}_{2}$ " o mecanismo de combustão foi reduzido de 47 reações e 16 substâncias para 15 reações e 9 substâncias; $(\operatorname{com} \eta(G, C) \approx 17.6)$; 
- para o bipropelente " $\mathrm{N}_{2} \mathrm{O}_{4}+\mathrm{C}_{2} \mathrm{H}_{8} \mathrm{~N}_{2}$ " o mecanismo de combustão foi reduzido de 80 reações e 26 substâncias para 27 reações e 15 substâncias, $(\operatorname{com} \eta(G, C) \approx 15.4)$.

\section{Agradecimentos}

Os autores agradecem ao Fundo de Pesquisas Fundamentais Russo (RFFI) e República do Tatarstan (projeto $N^{o} 15-48-02454$ /2017) pelo apoio financeiro.

\section{Referências}

[1] V. E. Alemassov, A. F. Dregalin, A. P. Tishin, V. A. Khudiakov and V. N. Kostin. Thermodynamic and Thermophysical Properties of Combustion Products. VINITI, Moscow, 1971.

[2] E. A. Barbour and R. K. Hanson. Chemical nonequilibrium, heat transfer, and friction in a detonation tube with nozzles, Journal of Propulsion and Power, 26:230-239, 2010.

[3] P. Glarborg, J. A. Miller and R. J. Kee. Kinetic modeling and sensitivity analysis of nitrogen oxide formation in well-stirred reactors, Combustion and Flame, 65:177-202, 1986.

[4] V. G. Krioukov, A. L. Abdullin and A. V. Demin. Difference schemes in computations for chemically non-equilibrium processes in the nozzles of rocket engines, Russian Aeronautica, 1:98-103, 2017.

[5] C. K. Law. Fuel options for next-generation chemical propulsion, AIAA Journal, 50:19-36, 2012.

[6] A. V. Lebedev, M. V. Okun' and A. E. Baranov. Simplification of kinetic mechanisms of physicochemical processes on the basis of combined mathematical methods, Chemical Physics and mMzoskopija, 13:43-52, 2011.

[7] P. Pepiot-Desjardins and H. Pitsch. An efficient error-propagation-based reduction method for large chemical kinetic mechanisms, Combustion and Flame,154:67-81, 2008.

[8] A. P. Spilimbergo, V. G. Krioukov and M. V. Nicandrova. Reduction of reaction mechanism by methods of DRGEP and of engagement. In Anais do Congresso Nacional de Matemática Aplicada e Computacional (XXVII CNMAC), São José dos Campos, São Paulo, Brasil, 2017.

[9] L. Tianfeng, J. Yiguang and C. K. Lam. Complex CSP for chemistry reduction and analysis, Combustion and Flame, 126:1445-1455, 2001. 